\title{
Intraoperative Hyperglycemia May be Associated with an Increased Risk of Myocardial Injury After Non-Cardiac Surgery in Diabetic Patients
}

\section{Sojin Kim}

Samsung Medical Center, Sungkyunkwan University School of Medicine Jungchan Park

Samsung Medical Center, Sungkyunkwan University School of Medicine

\section{Kwangmo Yang}

Samsung Medical Center, Sungkyunkwan University School of Medicine

Jin-ho Choi

Samsung Medical Center, Sungkyunkwan University School of Medicine

Kyunga Kim

Samsung Medical Center

Ji Dong Sung

Samsung Medical Center, Sungkyunkwan University School of Medicine Joonghyun Ahn

Samsung Medical Center

Seung-Hwa Lee ( $\sim$ shuaaa.lee@samsung.com )

Samsung Medical Center, Sungkyunkwan University School of Medicine

\section{Research Article}

Keywords: non-cardiac surgery, cardiac troponin, hyperglycemia

Posted Date: July 16th, 2021

DOI: https://doi.org/10.21203/rs.3.rs-696886/v1

License: (1) This work is licensed under a Creative Commons Attribution 4.0 International License. Read Full License 


\section{Abstract}

Background: Hyperglycemia in surgical candidates is associated with increased mortality and morbidity. We aimed to evaluate the effect of intraoperative blood glucose level on the incidence of myocardial injury after non-cardiac surgery (MINS) in diabetic patients.

Methods: Diabetic patients with available intraoperative blood glucose measurement during non-cardiac surgery were enrolled in this study. Based on the highest intraoperative blood glucose level, patients were stratified into two groups; the normal group (intraoperative peak glucose $<180 \mathrm{mg} / \mathrm{dL}$ ) and the hyperglycemia group (intraoperative peak glucose $\geq 180 \mathrm{mg} / \mathrm{dL}$ ). The primary outcome was the incidence of MINS, and secondary outcomes were in-hospital and 30-day mortalities.

Results: Of the 11,302 diabetic patients, 8,337 patients were in the normal group (73.8\%), and 2,965 patients were in the hyperglycemia group (26.2\%). After inverse probability weighting adjustment, MINS was significantly higher in the hyperglycemia group ( $24.0 \%$ vs $17.2 \%$; odds ratio [OR], 1.26 ; $95 \%$ confidence interval [Cl], $1.14-1.40 ; p<0.001)$. In addition, in-hospital and 30-day mortalities were also higher in the hyperglycemia group compared to the normal group (4.2\% vs $2.3 \%$, hazard ratio [HR], $1.39 ; 95 \% \mathrm{Cl}, 1.07$ $1.81 ; \mathrm{p}=0.01$, and $3.1 \%$ vs $1.8 \% ; \mathrm{HR}, 1.76 ; 95 \% \mathrm{Cl}, 1.31-2.36 ; \mathrm{p}<0.001$, respectively). Receiver-operating characteristic plots showed that the threshold of glucose level related to MINS was $149 \mathrm{mg} / \mathrm{dL}$.

Conclusion: Intraoperative hyperglycemia was associated with an increased MINS incidence and postoperative mortality in diabetic patients. Intraoperative blood glucose control may be helpful in preventing MINS.

\section{Introduction}

Hyperglycemia is associated with adverse outcomes in various clinical settings. ${ }^{1-3}$ During surgical procedures, blood glucose level elevates as a normal physiological response to surgical stimuli regardless of diabetes status, but diabetic patients undergo a higher risk of hyperglycemia owing to pathophysiologic changes such as impairment of vascular endothelium and cellular damage, leading to cardiovascular dysfunction, a major cause of mortality and morbidity. ${ }^{4,5}$ Numerous studies have recommended an adequate control of blood glucose level in diabetic patients undergoing surgical procedures, but mainly in cardiac surgeries ${ }^{6-8}$ or patients in critically ill states. ${ }^{9-11}$ Therefore, there is a paucity of data on the association between blood glucose level and cardiovascular events during non-cardiac surgical procedures.

Myocardial injury after non-cardiac surgery (MINS) has been recently accepted as the most common cardiovascular complication in surgical patients, with an incidence of $8-18 \%$ and regarded as an independent predictor of mortality. ${ }^{12,13}$ Previously, MINS was shown to be associated with preoperative hyperglycemia. ${ }^{14,15}$ However, considering that a transient elevation of glucose level in response to surgical stress is also associated with cardiovascular events, ${ }^{16}$ a separate analysis seems to be needed to assess intraoperative blood glucose level. Therefore, we aimed to evaluate whether intraoperative hyperglycemia 
is associated with the incidence of MINS in diabetic patients and estimated the cutoff value of the intraoperative peak blood glucose level that is associated with MINS. Our result may provide valuable evidence supporting the need for close monitoring of glucose level during non-cardiac surgery in diabetic patients.

\section{Methods}

\section{Study population and data collection}

In this retrospective observational study, we analyzed data from the SMC-TINCO registry (Samsung Medical Center Troponin in Non-cardiac Operation), which was approved by the Institutional Review Board at Samsung Medical Center (SMC 2019-08-048), Seoul, Korea, and the same committee also waived the requirement for informed written consent. Before patient enrollment, the cohort registration was done at https://cris.nih.go.kr (КСT0004244). The present study was conducted in accordance with the precepts of the Declaration of Helsinki and results are reported regarding the "Strengthening the Reporting of Observational Studies in Epidemiology" guidelines. ${ }^{17}$ This registry used de-identified data from the institutional electronic research data repository, Clinical Data Warehouse Darwin-C, and consists of 43,019 consecutive patients who had cardiac troponin (cTn I) levels measured before or within 30 days after noncardiac surgery between 2010 and 2019 at Samsung Medical Center. Demographic and perioperative data including medical, surgical, and anesthetic records were obtained from the registry. Mortality statistics were derived from the documents, and deaths outside of our institution were updated and verified with the National Population Registry of the Korea National Statistical Office.

The exclusion criteria for this study were as follows: 1) patients younger than 18 years old at the time of surgery, 2) patients with no postoperative cTn I measurements, 3) patients who had chest compression before the diagnosis of MINS, and 4) patients without available intraoperative blood glucose measurements. A total of 11,302 diabetic patients were engaged in the final analysis to examine the effect of transient hyperglycemia on diabetic patients.

Patients with a history of diabetes in their medical records or who used anti-diabetic medications at the time of admission were classified as diabetes. These patients were then stratified according to their elevation in intraoperative glucose level, $\geq 180 \mathrm{mg} / \mathrm{dL}$, which was obtained from arterial blood gas analysis during surgery. ${ }^{18-21}$

\section{Study outcomes and definitions}

The primary outcome was the incidence of MINS, defined as a peak cTn I level above the 99th percentile of the upper reference limit (URL) within 30 days after surgery. Following the diagnostic criteria, non-ischemic etiology that shows elevations of cTn I, such as pulmonary embolism, sepsis, cardioversion, atrial fibrillation or chronic elevation of cTn I were not considered MINS. ${ }^{12,13}$ Active cancer was defined as a histologic confirmation of malignancy within six months before surgery. ${ }^{22}$ High-risk surgery was grouped with a reported mortality risk greater than 5\%, according to the 2014 European Society of 
Cardiology/European Society of Anesthesiology (ESC/ESA) guidelines. ${ }^{23}$ The secondary outcomes were in-hospital mortality and 30-day mortality.

\section{Perioperative cTn I measurement and management}

At our hospital, cTn I measurement was recommended for patients with at least one of the following major cardiovascular risk factors: history of heart failure, ischemic heart disease, diabetes on insulin therapy, stroke or chronic kidney disease. In patients with minor risk factors, cTn I was measured at the discretion of the attending clinician considering recently suspected symptoms of ischemic disease or advance age. It was also recommended for those undergoing moderate- to high-risk surgery. An automated analyzer (Advia Centaur XP; Siemens Healthcare Diagnostics, Erlangen, Germany) with a highly sensitive cTn I immunoassay was used. According to the manufacturer, the lowest limit of detection was $6 \mathrm{ng} / \mathrm{L}$, and the 99th percentile URL was $40 \mathrm{ng} / \mathrm{L} .{ }^{24}$ Patients with elevated CTn I received consultation by a cardiologist and specialized management by an attending clinician.

\section{Intraoperative glucose measurement and management}

Patients undergoing surgery followed a strict NPO (nil per os; nothing by mouth) protocol for at least 8 hours of fasting from fatty food or meat before surgery. Therefore, the sole marker for glycemic status, which reflects acute fluctuation during the intraoperative period, was the fasting glucose. For patients with uncontrolled blood glucose elevation prior to surgery, endocrinology consultation was arranged. Oral antiglycemic drugs were withheld on the morning of surgery and restarted after surgery, when oral intake was permitted.

The management of diabetic patients followed the National Health Service $(\mathrm{NHS})^{18}$ and several other guidelines. ${ }^{19-21}$ We intended to maintain blood glucose level below $180 \mathrm{mg} / \mathrm{dL}(10 \mathrm{mmol} / \mathrm{L})$, and when significant hyperglycemia was detected, regular insulin was infused continuously or loaded as bolus injections at the discretion of an attending anesthesiologist. Glucose level was measured in arterial blood from a preplaced arterial line with a blood gas analyzer (RAPIDLab 1200 Blood Gas Analyzer; Siemens Healthcare, Erlangen, Germany) at $37^{\circ} \mathrm{C}$. An arterial line was indicated in case if hemodynamic instability or significant bleeding was anticipated. In addition to its role in continuous monitoring of invasive blood pressure and beat to beat variation in the patient's cardiovascular system, blood samples could be repeatedly drawn from this peripheral arterial line.

\section{Statistical analysis}

Differences were compared by the the Mann-Whitney test or the Student's t-test for continuous data and presented as the median values with interquartile range or mean \pm standard deviation. Categorical data are presented as the number (\%), and the Fisher's exact test or chi-square were applied to examine any association between categorical variables. The stratified logistic regression model was used to compare the incidence of MINS, and was reported as an adjusted odds ratio (OR) with a $95 \%$ confidence interval (Cl). Cox regression analysis was used to compare mortalities and reported as the hazard ratio (HR) with $95 \% \mathrm{Cl}$. To adjust for potential selection bias and imbalance in the patients' baseline characteristics, we 
employed an inverse probability weighted Cox regression model (IPW) ${ }^{25}$ To observe any interaction among relevant covariates, a subgroup analysis was conducted and presented as a forest plot. Pearson's correlation coefficient and receiver-operating characteristic (ROC) plots were conducted to assess the predictability of intraoperative glucose threshold in predicting MINS. The statistical power based on the sample size of the study size was calculated using Spearman's rank correlation. Sensitivity analyses of unmeasured confounders were performed. Statistical analyses were performed with R 4.0.2 (Vienna, Austria; http://www.R-project.org/) using the PowerSurvEpi (0.1.3) and Sensuc (6.2-0) packages. All data were evaluated using two-tailed t-tests, and statistical significance was assumed at $p$-values $<0.05$.

\section{Results}

Among 43,019 patients in the registry, the exclusion criteria were as follows: 19,297 patients without measurement of intraoperative glucose levels, 6,596 patients without postoperative cTn I measurements, 1,154 patients younger than 18 years old at the time of surgery and 46 patients who had chest compression prior to the diagnosis of MINS. The final study population consisted of 11,302 diabetic patients with available intraoperative blood glucose levels (Fig. 1).

Patients were divided into two groups according to the presence of significant intraoperative hyperglycemia (peak blood glucose level $\geq 180 \mathrm{mg} / \mathrm{dL}$ ): 8,337 patients $(73.8 \%$ ) without significant hyperglycemia (normal group), and 2,965 patients (26.2\%) with significant hyperglycemia (Table 1 ). Patients in the hyperglycemia group tended to be more hypertensive, current alcoholics, older, and tended to have active cancer, a history of preoperative in-hospital insulin administration, extracorporeal membranous oxygenation, ventilator and continuous renal replacement therapy. The percentage use of intraoperative insulin was also higher in the hyperglycemia group. Differences in operative variables included ESC/ESA operation risk, emergency operation requirement, operation duration, and the need for continuous infusion of inotropic agents or transfusion of packed red blood cells. The types of surgery each group has undergone are listed in Supplementary Table 1. 
Table 1

Baseline characteristics according to intraoperative blood glucose level.

\begin{tabular}{|c|c|c|c|c|c|c|}
\hline & \multirow{2}{*}{$\begin{array}{l}\text { Normal } \\
(n=8,337)\end{array}$} & \multirow{2}{*}{$\begin{array}{l}\text { Hyperglycemia } \\
(n=2,965)\end{array}$} & \multicolumn{2}{|c|}{ Before IPW } & \multicolumn{2}{|c|}{ After IPW } \\
\hline & & & $\begin{array}{l}\mathrm{P}- \\
\text { value }\end{array}$ & SMD & $\begin{array}{l}\mathrm{P}- \\
\text { value }\end{array}$ & SMD \\
\hline Intraoperative minimum BST* & $\begin{array}{l}120.3 \\
(25.8)\end{array}$ & $168.8(44.2)$ & $\begin{array}{l}< \\
0.001\end{array}$ & & $\begin{array}{l}< \\
0.001\end{array}$ & \\
\hline Intraoperative maximum BST* & $\begin{array}{l}134.8 \\
(25.6)\end{array}$ & $215.2(35.8)$ & $<.001$ & & $\dot{0} 001$ & \\
\hline Intraoperative insulin use & $\begin{array}{l}2925 \\
(35.1)\end{array}$ & $1251(42.2)$ & $\begin{array}{l}< \\
0.001\end{array}$ & $14.6 \%$ & 0.270 & $2.5 \%$ \\
\hline Age & $\begin{array}{l}61.0( \pm \\
13.7)\end{array}$ & $63.4( \pm 11.2)$ & $<.001$ & $19.8 \%$ & 0.046 & $4.7 \%$ \\
\hline Male & $\begin{array}{l}5235 \\
(62.8)\end{array}$ & $1822(61.5)$ & 0.203 & $2.8 \%$ & 0.243 & $2.7 \%$ \\
\hline Hypertension & $\begin{array}{l}4733 \\
(56.8)\end{array}$ & $1908(64.4)$ & $\begin{array}{l}< \\
0.001\end{array}$ & $15.6 \%$ & 0.428 & $1.9 \%$ \\
\hline Chronic kidney disease & $637(7.6)$ & $252(8.5)$ & 0.147 & $3.2 \%$ & 0.832 & $0.5 \%$ \\
\hline Current alcoholic & $\begin{array}{l}1753 \\
(21.0)\end{array}$ & $515(17.4)$ & $\begin{array}{l}< \\
0.001\end{array}$ & $9.3 \%$ & 0.917 & $0.3 \%$ \\
\hline Active cancer & $\begin{array}{l}4408 \\
(52.9)\end{array}$ & $1801(60.7)$ & $<0.001$ & $15.9 \%$ & 0.505 & $1.6 \%$ \\
\hline \multicolumn{7}{|l|}{ Previous disease } \\
\hline Stroke & $573(6.9)$ & $196(6.6)$ & 0.656 & $1.0 \%$ & 0.891 & $0.3 \%$ \\
\hline Coronary artery disease & $\begin{array}{l}1191 \\
(14.3)\end{array}$ & $470(15.9)$ & 0.042 & $4.4 \%$ & 0.641 & $1.1 \%$ \\
\hline Heart failure & $194(2.3)$ & $43(1.5)$ & 0.005 & $6.4 \%$ & 0.298 & $2.6 \%$ \\
\hline Arrythmia & $559(6.7)$ & $160(5.4)$ & 0.014 & $5.5 \%$ & 0.218 & $3.2 \%$ \\
\hline Heart valvular disease & $81(1.0)$ & $19(0.6)$ & 0.124 & $3.7 \%$ & 0.644 & $1.3 \%$ \\
\hline \multicolumn{7}{|l|}{ Preoperative in-hospital care } \\
\hline Insulin use & $\begin{array}{l}7508 \\
(90.1)\end{array}$ & $2788(94.0)$ & $<.001$ & $14.7 \%$ & 0.026 & $5.9 \%$ \\
\hline
\end{tabular}

Data are presented as $n(\%)$, mean ( \pm standard deviation) or median (interquertile range).

IPW, inverse probability weighting; SMD, standardized mean difference; BST,blood suger test; ECMO, extracorporeal membranous oxygenation; ESC, European Society of Cardiology; ESA, European Society of Anaesthesiology. 


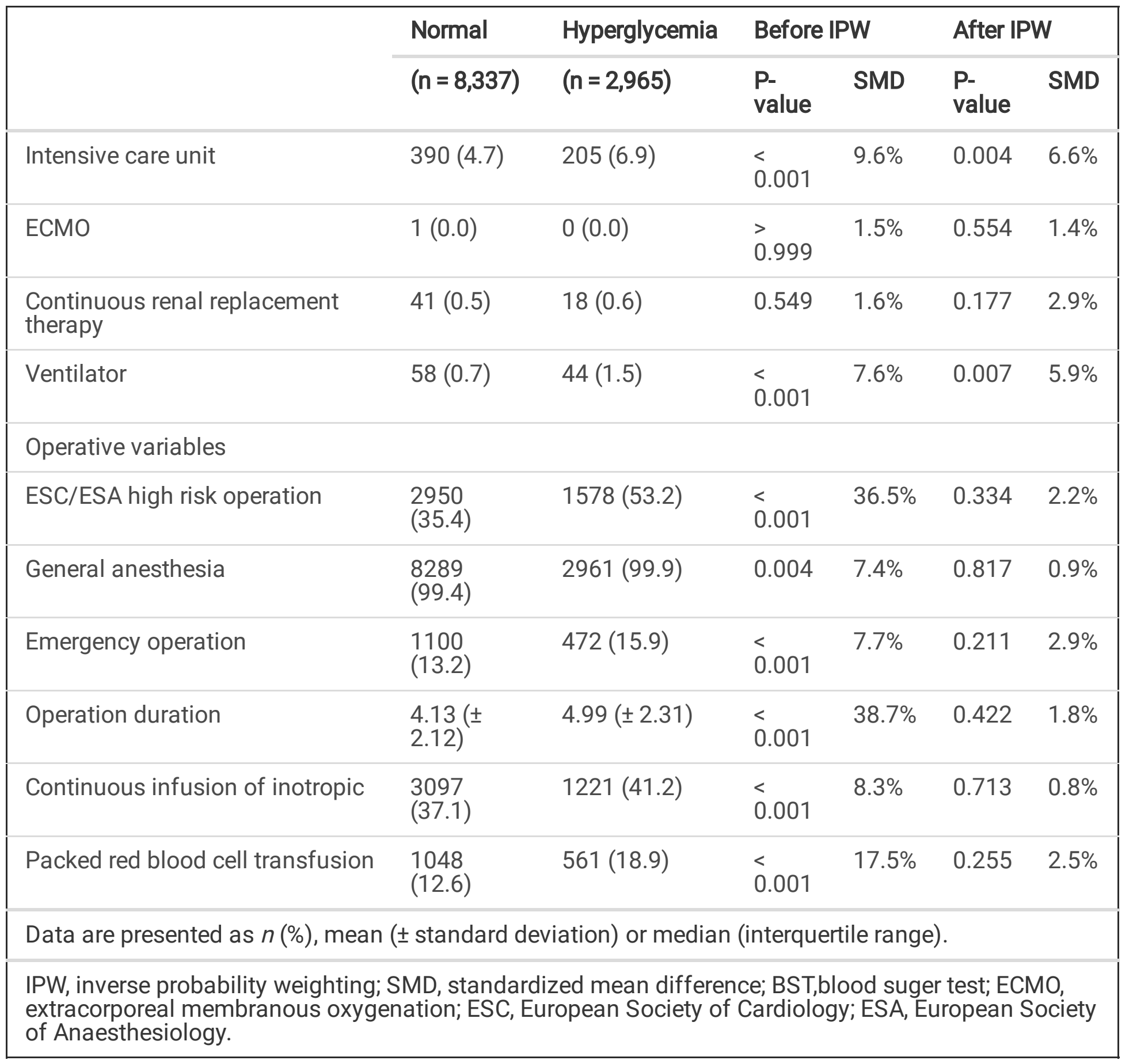

Of the 3,193 patients with postoperative cTn elevation, 110 patients were diagnosed with a non-ischemic cause; therefore, a total of 2,147 (19.0\%) patients were diagnosed with MINS. The incidence of MINS was significantly higher in the hyperglycemia group after adjustment with multivariable analysis $(24.0 \% \mathrm{vs}$ 17.2\%; OR, 1.29; 95\% Cl, 1.15-1.43; $P<0.001$ ) (Table 2). Our results remained significant after IPW adjustment, and the incidence of MINS was significantly associated with hyperglycemia (OR, 1.26; $95 \% \mathrm{Cl}$, 1.14-1.40; $P<0.001$ ) (Table 2). Our study also revealed that the risk of in-hospital mortality and 30-day mortality was significantly higher in the hyperglycemia group. (4.2\% vs $2.3 \%$; adjusted $\mathrm{OR}, 1.36 ; 95 \% \mathrm{Cl}$, $0.74-1.08 ; P=0.01$, and $3.1 \%$ vs $1.8 \%$; adjusted $\mathrm{OR}, 1.71 ; 95 \% \mathrm{Cl}, 1.30-2.25 ; P<0.001$, respectively) (Table 2). These results were consistent after IPW adjustment (OR, 1.39; 95\% Cl, 1.07-1.81; $P=0.01$ for inhospital mortality and $\mathrm{OR}, 1.76 ; 95 \% \mathrm{Cl}, 1.31-2.36 ; P<0.001$ for 30-day mortality, respectively). Subgroup 
analysis revealed that the association between hyperglycemia and MINS was confounded by hypertension, chronic renal failure, high-risk surgical operation, and active cancer. ( $P$ for interaction $<0.001$, $P<0.001, P=0.001$, and $P=0.007$, respectively) (Fig. 2). The incidence of MINS was significantly increased by hyperglycemia only in patients without hypertension (OR 1.74; 95\% $\mathrm{Cl}, 1.46-2.07, P<0.001$ ), without chronic renal failure patients (OR 1.36; $95 \% \mathrm{Cl}, 1.22-1.52, P=0.001)$, without active cancer (OR $1.45 ; 95 \% \mathrm{Cl}, 1.27-1.67, P<0.001)$, and who were not undergoing high-risk operation. (OR $1.53 ; 95 \% \mathrm{Cl}$, 1.34-1.75, $P<0.001)$. The spline curve for the log odds ratio of intraoperative blood glucose level versus the risk of MINS was plotted (Fig. 3). The estimated threshold for intraoperative glucose level related to MINS was $149 \mathrm{mg} / \mathrm{dL}$, by means of ROC analysis, and the area under the ROC curve was 0.57 . The sensitivity and specificity of $59.0 \%$ and $51.4 \%$, respectively, were obtained (Fig. 4 ). The power analysis of the sample size was 0.99 when the OR for MINS was 1.3 , and 0.95 when the OR for MINS was 1.2. The effect of an unmeasured confounder was estimated with an assumed prevalence of $40 \%$, and the observed associations were all significant (Supplementary Table 2).

Table 2

The incidence of myocardial after non-cardiac surgery and mortality according to preoperative blood glucose level.

\begin{tabular}{|c|c|c|c|c|c|c|c|c|}
\hline & \multirow{2}{*}{$\begin{array}{l}\text { Normal } \\
(n= \\
8,337)\end{array}$} & \multirow{2}{*}{$\begin{array}{l}\text { Hyperglycemia } \\
(n=2,965)\end{array}$} & \multicolumn{2}{|c|}{ Univariable analysis } & \multicolumn{2}{|c|}{$\begin{array}{l}\text { Multivariable } \\
\text { analysis }\end{array}$} & \multicolumn{2}{|c|}{ After IPW } \\
\hline & & & $\begin{array}{l}\text { Unadjusted } \\
\text { OR/HR } \\
(95 \% \mathrm{Cl})\end{array}$ & $\begin{array}{l}\text { p- } \\
\text { value }\end{array}$ & $\begin{array}{l}\text { Adjusted } \\
\text { OR/HR } \\
(95 \% \mathrm{Cl})\end{array}$ & $\begin{array}{l}\mathrm{p}- \\
\text { value }\end{array}$ & $\begin{array}{l}\text { OR/HR } \\
(95 \% \\
\text { Cl) }\end{array}$ & $\begin{array}{l}\mathrm{p}- \\
\text { value }\end{array}$ \\
\hline MINS & $\begin{array}{l}1434 \\
(17.2)\end{array}$ & $713(24.0)$ & $\begin{array}{l}1.52 \\
(1.38- \\
1.69)\end{array}$ & $\begin{array}{l}<.001 \\
0.001\end{array}$ & $\begin{array}{l}1.29 \\
(1.15- \\
1.43)\end{array}$ & $\begin{array}{l}<.001 \\
0.00\end{array}$ & $\begin{array}{l}1.26 \\
(1.14- \\
1.40)\end{array}$ & $\begin{array}{l}< \\
0.001\end{array}$ \\
\hline $\begin{array}{l}\text { In- } \\
\text { hospital } \\
\text { mortality }\end{array}$ & $\begin{array}{l}191 \\
(2.3)\end{array}$ & $125(4.2)$ & $\begin{array}{l}1.39 \\
(1.11- \\
1.74)\end{array}$ & 0.005 & $\begin{array}{l}1.36 \\
(0.74- \\
1.08)\end{array}$ & 0.01 & $\begin{array}{l}1.39 \\
(1.07- \\
1.81)\end{array}$ & 0.01 \\
\hline $\begin{array}{l}\text { 30-day } \\
\text { mortality }\end{array}$ & $\begin{array}{l}147 \\
(1.8)\end{array}$ & $93(3.1)$ & $\begin{array}{l}1.80 \\
(1.39- \\
2.33)\end{array}$ & $\begin{array}{l}< \\
0.001\end{array}$ & $\begin{array}{l}1.71 \\
(1.30- \\
2.25)\end{array}$ & $\begin{array}{l}< \\
0.001\end{array}$ & $\begin{array}{l}1.76 \\
(1.31- \\
2.36)\end{array}$ & $\begin{array}{l}< \\
0.001\end{array}$ \\
\hline
\end{tabular}

\section{Discussion}

This study demonstrated that significant hyperglycemia in diabetic patients during non-cardiac surgery confers an increased MINS and mortality incidence. The estimated threshold for intraoperative blood glucose level related to MINS was $149 \mathrm{mg} / \mathrm{dL}$. Our findings suggest that managing for an adequate intraoperative blood glucose level may be beneficial for the prevention of MINS.

Perioperative hyperglycemia is common as a result of transient body glucose elevation in response to physiological stress among patients undergoing surgery. The stress of surgery and anesthesia causes the release of counter-regulatory hormones, leading to reduced insulin production and increased lipolysis. ${ }^{4}$ 
Sustained hyperglycemia triggers insulin resistance by impairment of the insulin signal transduction pathway, resulting in cardiovascular events such as myocardial injury. ${ }^{14,26}$ Perioperative hyperglycemia is also associated with numerous other adverse outcomes including longer postoperative hospital duration, surgical site infection, higher overall morbidity, and mortality. ${ }^{1,27}$ By inducing acute changes in cellular metabolism, such as an increase in polyol pathway activation and hexosamine pathway flux, activation of protein kinase $\mathrm{C}$, formation of advanced glycation end products, and hyperglycemia leads to tissue damage, vascular inflammation, fibrosis, and injury by overproduction of reactive oxygen species. ${ }^{28}$

Our study focused on the effect of transient hyperglycemia superimposed on diabetic patients who were already at risk for various adverse clinical outcomes and complications related to diabetes itself. According to our result, intraoperative hyperglycemia was associated with an increased risk of MINS. This result is consistent with our previous finding that MINS was associated with preoperative glucose level rather than hemoglobin A1c level, which reflects more of a long-term glucose status. ${ }^{15}$ Both results stress the association between short-term glycemic control and the incidence of MINS.

The mediation of reactive oxygen species could explain the mechanism behind the association between hyperglycemia and MINS. The imbalance between free radical generation and elimination plays a key role in the pathophysiology of myocardial injury. ${ }^{28}$ During the perioperative period, oxidative stress due to hyperglycemia and insulin resistance contributes to the cellular structure and functions of diabetic myocardium. ${ }^{29,30}$ Furthermore, surgical hypercoagulability, bleeding, and inflammation can also predispose patients to ischemic injuries. ${ }^{31}$

In this study, we divided the patients according to intraoperative blood glucose level above $180 \mathrm{mg} / \mathrm{dL}$, while the estimated threshold associated with MINS incidence was $149 \mathrm{mg} / \mathrm{dL}$. Stress hyperglycemia has been consistently related to adverse outcomes in both diabetic and non-diabetic patients. ${ }^{27,32}$ In a previous study, even modest glucose elevation $(140-179 \mathrm{mg} / \mathrm{dL})$ was strongly related to mortality only in non-diabetic patients, but more severe hyperglycemia (glucose $>180 \mathrm{mg} / \mathrm{dL}$ ) was a risk factor for mortality among diabetic patients ${ }^{2}$. Because the purpose of this study was to evaluate the effect of stress-induced hyperglycemia in diabetic patients, we stratified patients into the hyperglycemia group even with a single intraoperative glucose level over $180 \mathrm{mg} / \mathrm{dL}$. Therefore, our results also need to be validated in patients without diabetes. Of note, the subgroup analysis showed that the observed association between hyperglycemia and MINS was not significant in patients with hypertension, chronic renal failure, active cancer, and for those undergoing high-risk surgery. In addition, the threshold of intraoperative blood glucose associated with MIN had an area under the ROC curve of 0.57 with relatively low sensitivity and specificity; therefore, further investigation regarding more precise cutoff value for intraoperative blood glucose level is required.

For intraoperative blood glucose control, the 2019 ADA guidelines recommend a range between $80-$ $200 \mathrm{mg} / \mathrm{dL}$ (4.4-10.0mmol/L), ${ }^{33}$ based on numerous studies suggesting tight glycemic control during surgery. ${ }^{6,34}$ On the other hand, conflicting results have also been reported, suggesting that aggressive intraoperative treatment of hyperglycemia did not improve outcome; in some instances, such treatment 
actually increased mortality and postoperative stroke. ${ }^{16,30}$ For the patients of the present study, regular insulin was used to treat hyperglycemia during the surgical period. The use of regular insulin is known to have anti-inflammatory, anti-thrombotic, and anti-atherogenic properties with a favorable effect on

myocardium. ${ }^{35,36}$ However, an accurate blood glucose target during surgery was not identified in this study and therefore needs further investigation.

There are several limitations to be addressed. Due to the nature of a retrospective study, perioperative cTn I and intraoperative blood glucose levels were not measured in all the patients undergoing non-cardiac surgery; In addition, as they were selectively performed selectively in high-risk patients, our results may have been exaggerated due to selection bias. Second, a unifying treatment protocol for perioperative hyperglycemic management was absent. Anesthesia was provided by many different anesthesiologists; therefore, a therapeutic approach to hyperglycemia such as the dose of insulin given and at which glucose level the treatment initiation has taken place may have varied, thereby possibly influencing the results. Lastly, our study could not fully address the question of adequate blood glucose level or whether treatment of hyperglycemia with insulin may reduce the incidence of MINS or mortality. Despite these limitations, this study showed that MINS in diabetic patients was associated with intraoperative blood glucose level, highlighting the importance of glycemic control during surgical procedures.

\section{Conclusion}

Intraoperative hyperglycemia (fasting glucose $\geq 180 \mathrm{mg} / \mathrm{dL}$ ) appears to be associated with an increased risk of MINS in diabetic patients. Continuous monitoring and management of intraoperative glucose may potentially protect diabetic patients from developing MINS.

\section{Declarations}

\section{Authors' contributions:}

Substantial contributions to the conception or design of the work: SK, JP, SHL

Acquisition, analysis: KK, JA

Interpretation of data: SK, JP, SHL, KY, JhC

Drafting the manuscript: SK, JP

Revising the manuscript critically for important intellectual content: KY, JhC, KK, JDS, JA, SHL

The first two authors contributed equally to this work (SK, JP)

All authors reviewed the manuscript. 


\section{Disclosures:}

None.

\section{Competing Interests:}

The authors have declared that no competing interests exist.

\section{Funding:}

The authors received no specific funding for this work.

\section{References}

1. Thompson, B. M., Stearns, J. D., Apsey, H. A., Schlinkert, R. T. \& Cook, C. B. Perioperative Management of Patients with Diabetes and Hyperglycemia Undergoing Elective Surgery. Curr Diab Rep, 16, 2 https://doi.org/10.1007/s11892-015-0700-8 (2016).

2. Palermo, N. E., Gianchandani, R. Y., McDonnell, M. E. \& Alexanian, S. M. Stress Hyperglycemia During Surgery and Anesthesia: Pathogenesis and Clinical Implications. Curr Diab Rep, 16, 33 https://doi.org/10.1007/s11892-016-0721-y (2016).

3. Galindo, R. J., Fayfman, M. \& Umpierrez, G. E. Perioperative Management of Hyperglycemia and Diabetes in Cardiac Surgery Patients. Endocrinol Metab Clin North Am, 47, 203-222 https://doi.org/10.1016/j.ecl.2017.10.005 (2018).

4. Duggan, E. W., Carlson, K. \& Umpierrez, G. E. Perioperative Hyperglycemia Management: An Update., 126, 547-560 https://doi.org/10.1097/ALN.0000000000001515 (2017).

5. Carson, J. L. et al. Diabetes mellitus increases short-term mortality and morbidity in patients undergoing coronary artery bypass graft surgery. J Am Coll Cardiol, 40, 418-423 https://doi.org/10.1016/s0735-1097(02)01969-1 (2002).

6. Lazar, H. L. et al. Tight glycemic control in diabetic coronary artery bypass graft patients improves perioperative outcomes and decreases recurrent ischemic events., 109, 1497-1502 https://doi.org/10.1161/01.CIR.0000121747.71054.79 (2004).

7. Oezkur, M. et al. Chronic hyperglycemia is associated with acute kidney injury in patients undergoing CABG surgery-a cohort study. BMC Cardiovasc Disord, 15, 41 https://doi.org/10.1186/s12872-0150028-y (2015).

8. Ouattara, A. et al. Poor intraoperative blood glucose control is associated with a worsened hospital outcome after cardiac surgery in diabetic patients., 103, 687-694 https://doi.org/10.1097/00000542200510000-00006 (2005).

9. Bower, W. F. et al. Peri-operative hyperglycemia: a consideration for general surgery? Am J Surg, 199, 240-248 https://doi.org/10.1016/j.amjsurg.2009.04.010 (2010). 
10. Clodi, M., Resl, M., Abrahamian, H., Foger, B. \& Weitgasser, R. [Treatment of hyperglycemia in adult, critically ill patients (Update 2019)]. Wien Klin Wochenschr, 131, 218-220 https://doi.org/10.1007/s00508-019-1486-5 (2019).

11. Gokhale, V., Batra, T., Shinde, S. S., Gulati, S. \& Kakrani, A. L. Glucose Monitoring in Critically III: Is Absence of "Stress Hyperglycemia" a Cause for Concern? J Assoc Physicians India, 67, 43-46 (2019).

12. Vascular Events In Noncardiac Surgery Patients Cohort Evaluation Study Investigators. et al. Association between postoperative troponin levels and 30-day mortality among patients undergoing noncardiac surgery. JAMA, 307, 2295-2304 https://doi.org/10.1001/jama.2012.5502 (2012).

13. Botto, F. et al. Myocardial injury after noncardiac surgery: a large, international, prospective cohort study establishing diagnostic criteria, characteristics, predictors, and 30-day outcomes., 120, 564-578 https://doi.org/10.1097/ALN.0000000000000113 (2014).

14. Punthakee, Z. et al. Association of preoperative glucose concentration with myocardial injury and death after non-cardiac surgery (GlucoVISION): a prospective cohort study. Lancet Diabetes Endocrinol, 6, 790-797 https://doi.org/10.1016/S2213-8587(18)30205-5 (2018).

15. Park, J. et al. Associations Between Preoperative Glucose and Hemoglobin A1c Level and Myocardial Injury After Noncardiac Surgery. J Am Heart Assoc, 10, e019216 https://doi.org/10.1161/JAHA.120.019216 (2021).

16. Buchleitner, A. M., Martinez-Alonso, M., Hernandez, M., Sola, I. \& Mauricio, D. Perioperative glycaemic control for diabetic patients undergoing surgery. Cochrane Database Syst Rev, CD007315, https://doi.org/10.1002/14651858.CD007315.pub2 (2012).

17. von Elm, E. et al. The Strengthening the Reporting of Observational Studies in Epidemiology (STROBE) Statement: guidelines for reporting observational studies. Int J Surg, 12, 1495-1499 https://doi.org/10.1016/j.ijsu.2014.07.013 (2014).

18. Dhatariya, K. et al. NHS Diabetes guideline for the perioperative management of the adult patient with diabetes. Diabet Med, 29, 420-433 https://doi.org/10.1111/j.1464-5491.2012.03582.x (2012).

19. Joshi, G. P. et al. Society for Ambulatory Anesthesia consensus statement on perioperative blood glucose management in diabetic patients undergoing ambulatory surgery. Anesth Analg, 111, 13781387 https://doi.org/10.1213/ANE.0b013e3181f9c288 (2010).

20. Moghissi, E. S. et al. American Association of Clinical Endocrinologists and American Diabetes Association consensus statement on inpatient glycemic control., 32, 1119-1131 https://doi.org/10.2337/dc09-9029 (2009).

21. Lazar, H. L. et al. The Society of Thoracic Surgeons practice guideline series: Blood glucose management during adult cardiac surgery. Ann Thorac Surg, 87, 663-669 https://doi.org/10.1016/j.athoracsur.2008.11.011 (2009).

22. Lee, A. Y. Y. et al. Tinzaparin vs Warfarin for Treatment of Acute Venous Thromboembolism in Patients With Active Cancer: A Randomized Clinical Trial. JAMA, 314, 677-686 https://doi.org/10.1001/jama.2015.9243 (2015). 
23. Kristensen, S. D. et al. 2014 ESC/ESA Guidelines on non-cardiac surgery: cardiovascular assessment and management: The Joint Task Force on non-cardiac surgery: cardiovascular assessment and management of the European Society of Cardiology (ESC) and the European Society of Anaesthesiology (ESA). Eur Heart J 35, 2383-2431, http://doi.org/10.1093/eurheartj/ehu282 (2014).

24. Mahajan, V. S. \& Jarolim, P. How to interpret elevated cardiac troponin levels., 124, 2350-2354 https://doi.org/10.1161/CIRCULATIONAHA.111.023697 (2011).

25. Austin, P. C. \& Stuart, E. A. Moving towards best practice when using inverse probability of treatment weighting (IPTW) using the propensity score to estimate causal treatment effects in observational studies. Stat Med, 34, 3661-3679 https://doi.org/10.1002/sim.6607 (2015).

26. Tomas, E. et al. Hyperglycemia and insulin resistance: possible mechanisms. Ann NY Acad Sci, 967, 43-51 https://doi.org/10.1111/j.1749-6632.2002.tb04262.x (2002).

27. Di Luzio, R. et al. Stress Hyperglycemia and Complications Following Traumatic Injuries in Individuals With/Without Diabetes: The Case of Orthopedic Surgery. Diabetes Metab Syndr Obes, 13, 9-17 https://doi.org/10.2147/DMSO.S225796 (2020).

28. Brownlee, M. The pathobiology of diabetic complications: a unifying mechanism., 54, 1615-1625 https://doi.org/10.2337/diabetes.54.6.1615 (2005).

29. Ansley, D. M. \& Wang, B. Oxidative stress and myocardial injury in the diabetic heart. J Pathol, 229, 232-241 https://doi.org/10.1002/path.4113 (2013).

30. Duncan, A. E. Hyperglycemia and perioperative glucose management. Curr Pharm Des, 18, 61956203 https://doi.org/10.2174/138161212803832236 (2012).

31. Nielsen, V. G. \& Asmis, L. M. Hypercoagulability in the perioperative period. Best Pract Res Clin Anaesthesiol, 24, 133-144 https://doi.org/10.1016/j.bpa.2009.09.012 (2010).

32. Frisch, A. et al. Prevalence and clinical outcome of hyperglycemia in the perioperative period in noncardiac surgery., 33, 1783-1788 https://doi.org/10.2337/dc10-0304 (2010).

33. American Diabetes Association. 15. Diabetes Care in the Hospital: Standards of Medical Care in Diabetes-2020. Diabetes Care 43, S193-S202, http://doi.org/10.2337/dc20-S015 (2020).

34. Kwon, S. et al. Importance of perioperative glycemic control in general surgery: a report from the Surgical Care and Outcomes Assessment Program. Ann Surg, 257, 8-14 https://doi.org/10.1097/SLA.0b013e31827b6bbc (2013).

35. Dandona, P., Aljada, A. \& Mohanty, P. The anti-inflammatory and potential anti-atherogenic effect of insulin: a new paradigm., 45, 924-930 https://doi.org/10.1007/s00125-001-0766-5 (2002).

36. Iliadis, F., Kadoglou, N. \& Didangelos, T. Insulin and the heart. Diabetes Res Clin Pract, 93 Suppl (1), S86-91 https://doi.org/10.1016/S0168-8227(11)70019-5 (2011).

\section{Figures}




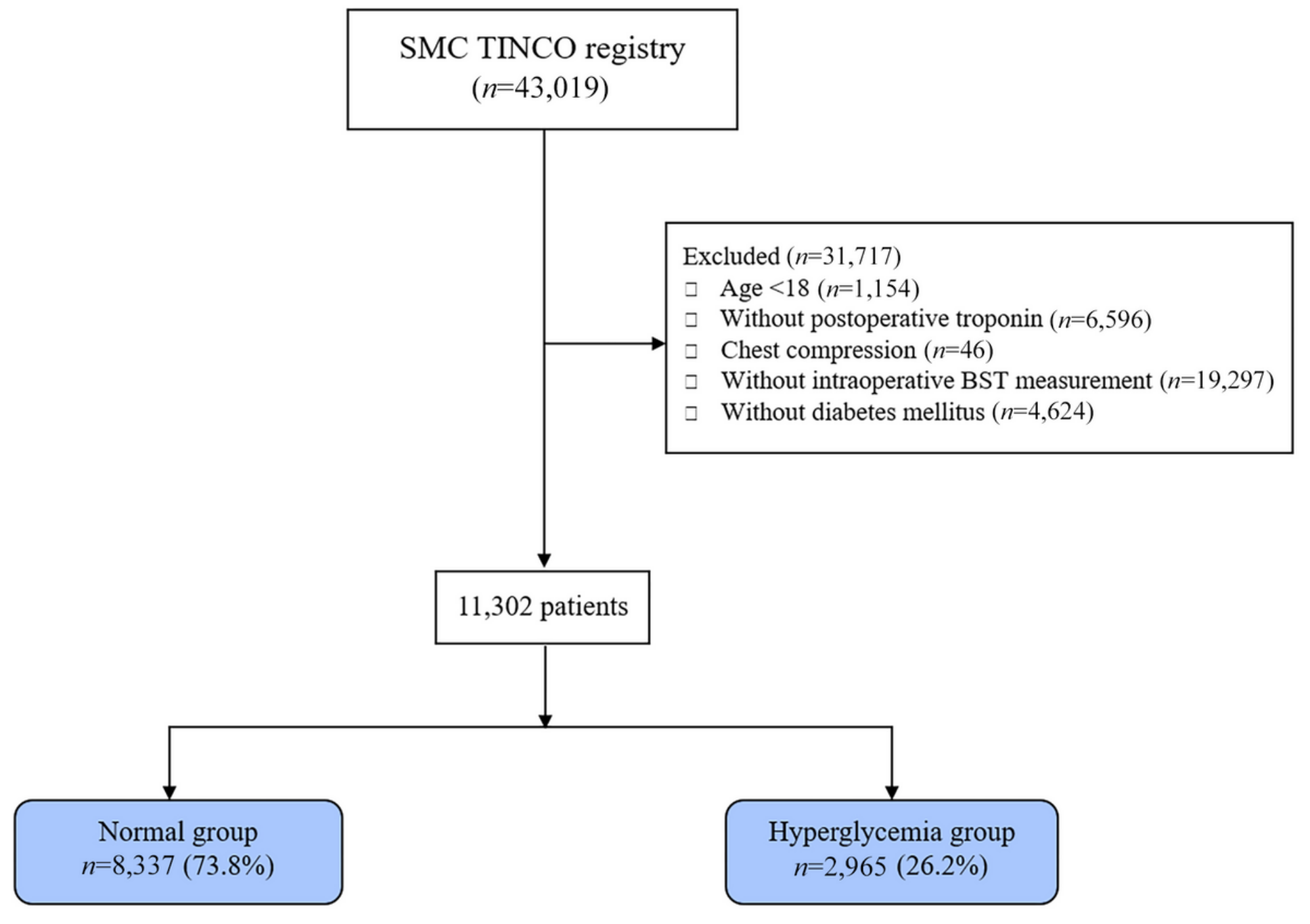

Figure 1

Flowchart of study patients. 


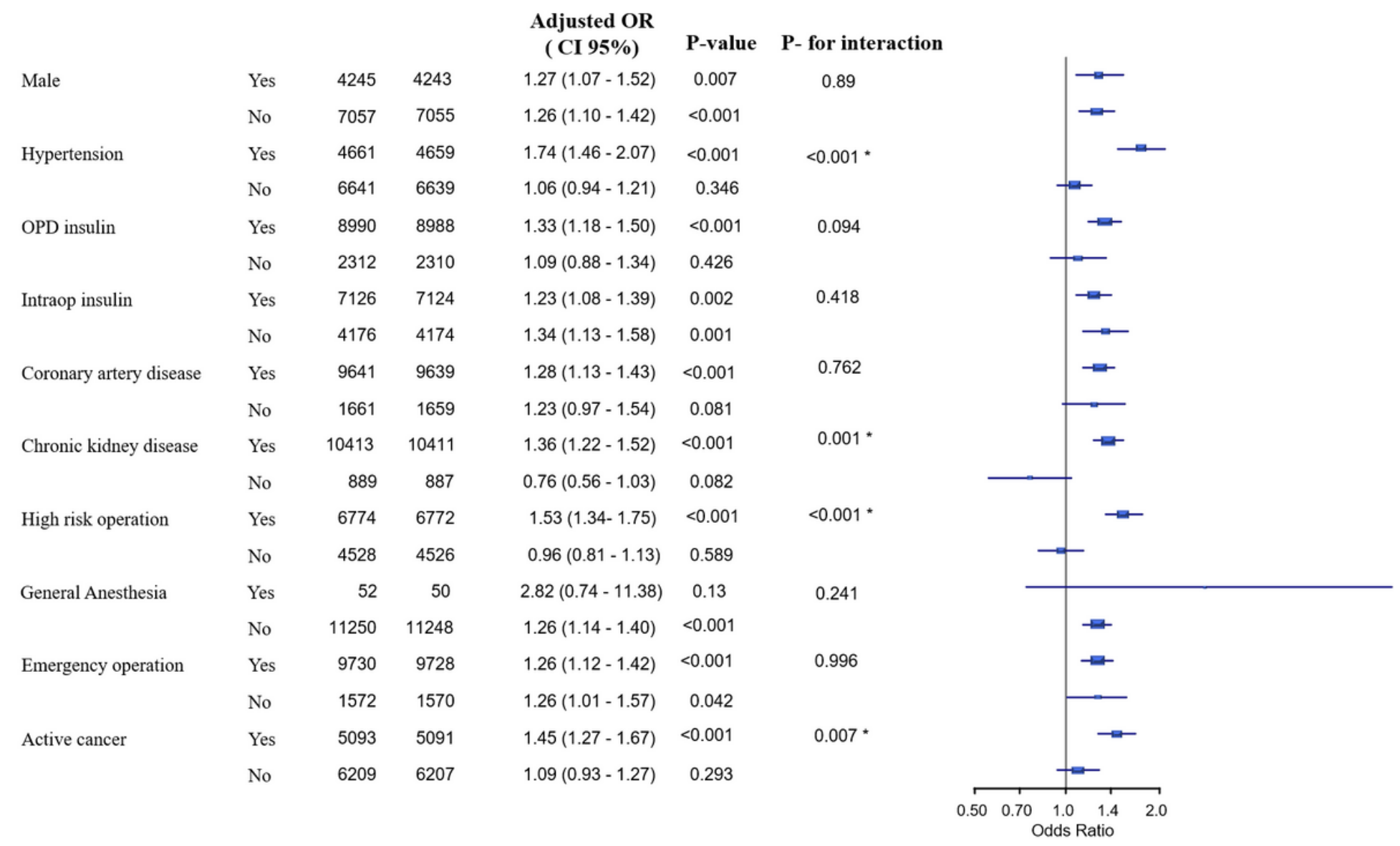

Figure 2

Forest plot from subgroup analysis showing the association between significant hyperglycemia and the risk of MINS. 


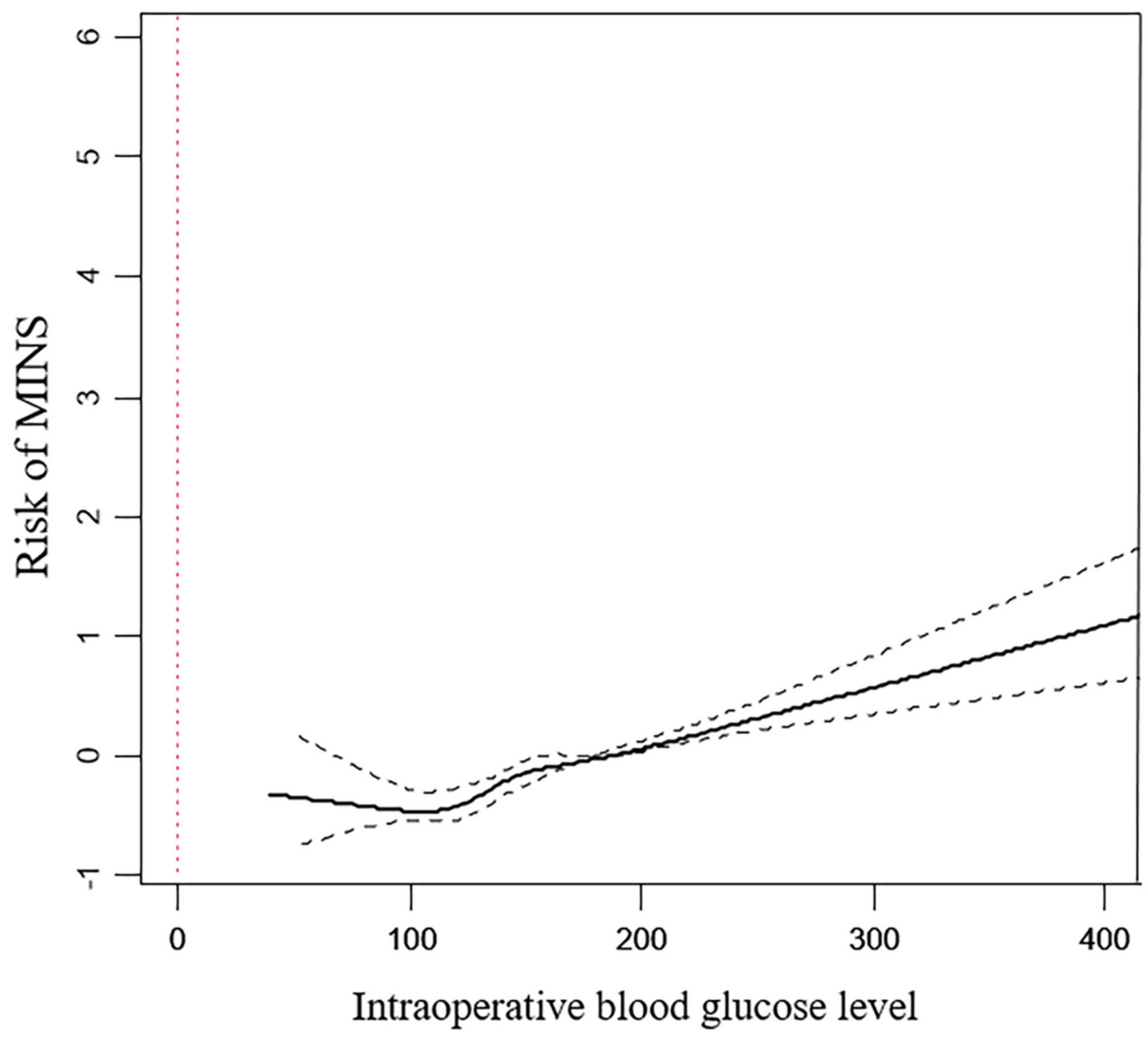

Figure 3

Spline curve for the log odds ratio of intraoperative blood glucose level versus the risk of MINS. 


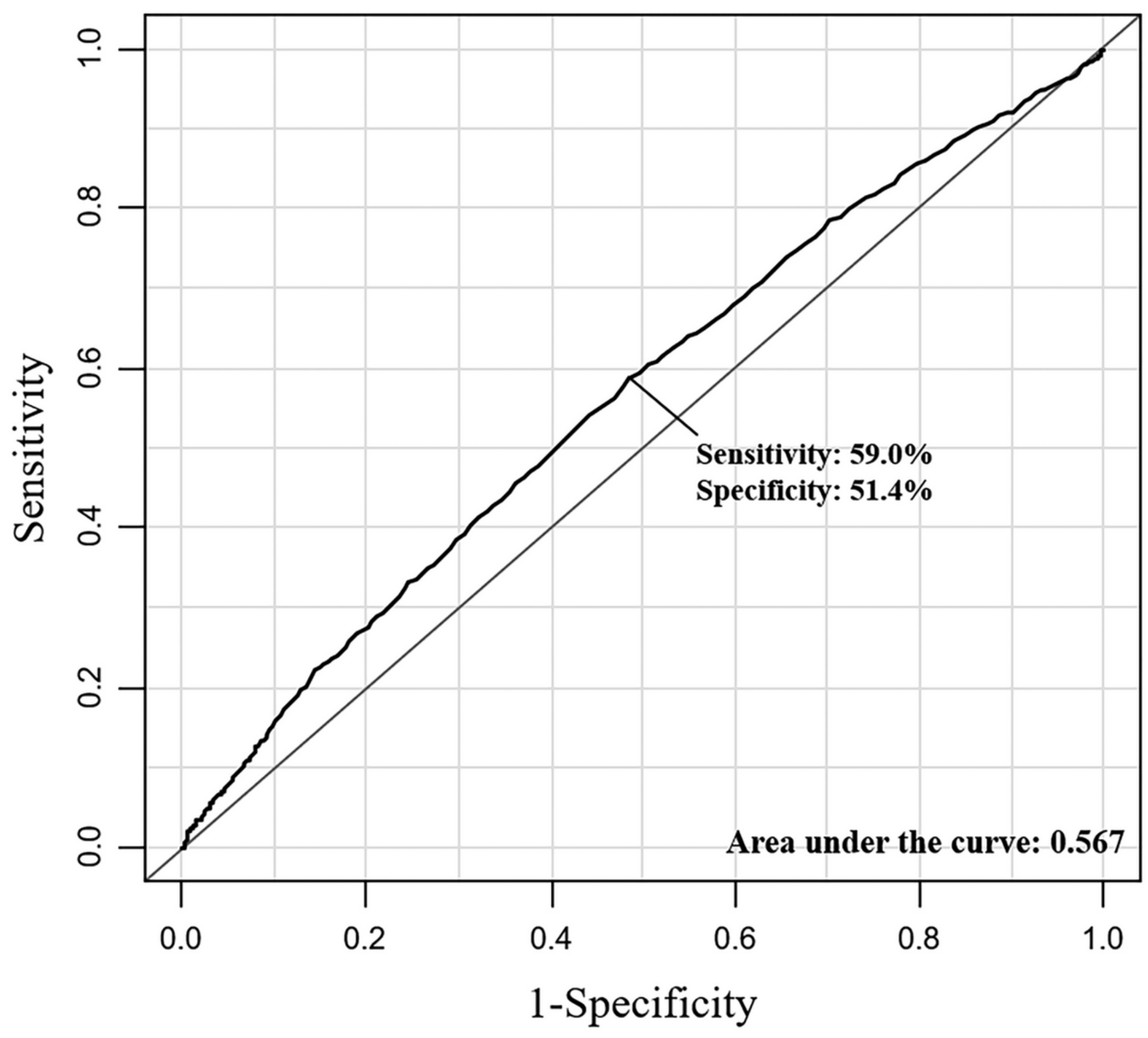

Figure 4

Receiver-operating characteristic plots to estimate the threshold of intraoperative blood glucose level associated with MINS.

\section{Supplementary Files}

This is a list of supplementary files associated with this preprint. Click to download.

- Hyperglycemiasupplementary.pdf 